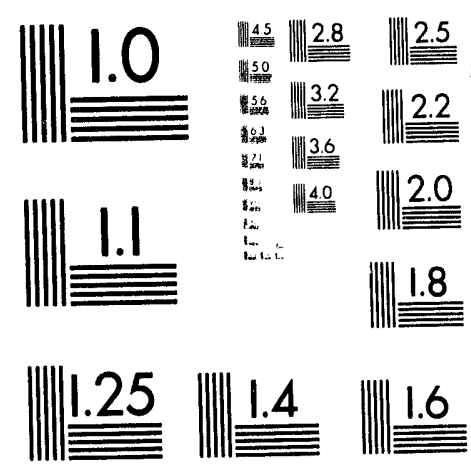



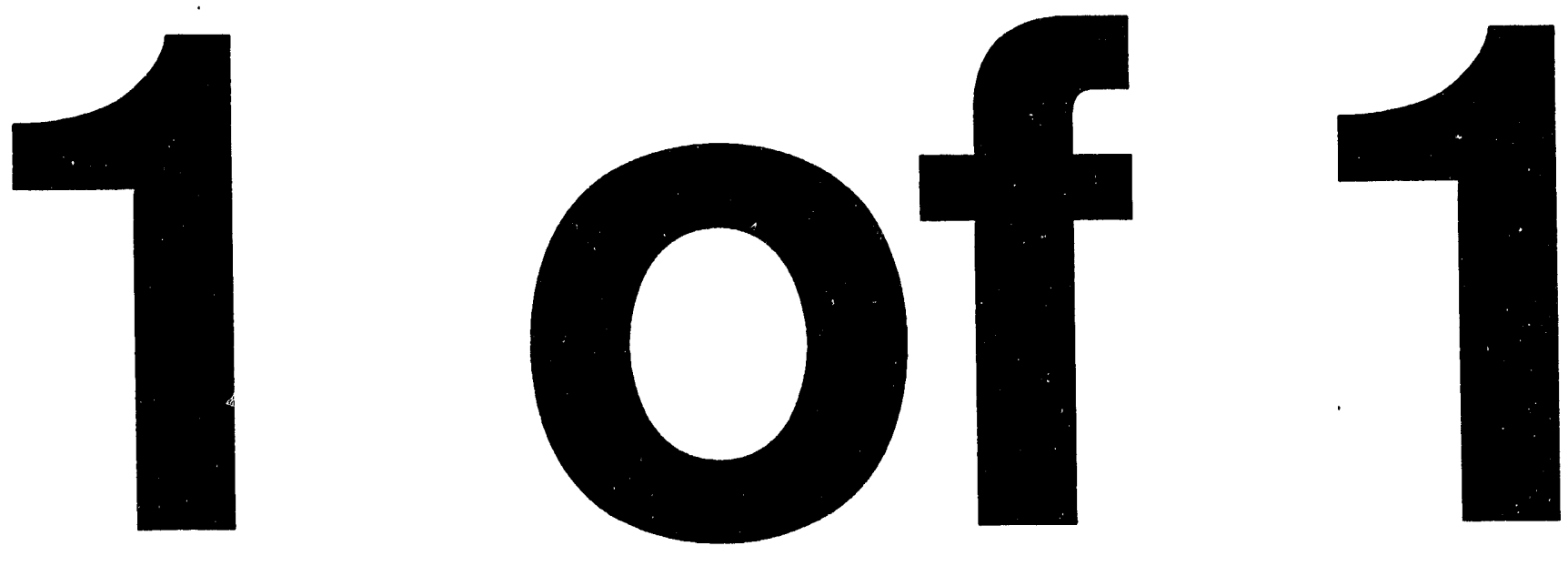


\title{
THE USE OF DIFFRACTION EFFICIENCY THEORY IN THE DESIGN OF SOFT X-RAY MONOCHROMATORS
}

\author{
H.A. Padmore, V. Martynov, K. Hollis
}

\author{
Advanced Light Source \\ Accelerator and Fusion Research Division \\ Lawrence Berkeley Laboratory \\ University of California \\ Berkeley,CA 94720
}

August 23, 1993

Paper Presented at the 8th National Conference on Synchrotron Radiation Instrumentation, Gaithersburg, Maryland, USA, 8/23/93-8/26/93. 
Presented at the Synchrotron Radiation Instrumentation meeting in Gaithersburg, August 23rd - 26th, 1993.

The use of diffraction efficiency theory in the design of soft x-ray monochromators

\author{
H. A. Padmore, 1 V. Martynov, 2,3 and K. Hollis 2,4 \\ ${ }^{1}$ Advanced Light Source, Accelerator and Fusion Research Division, \\ Lawrence Berkeley Laboratory, Berkeley, CA 94720, USA \\ 2 Daresbury Laboratory, Daresbury, Warrington WA4 4AD, UK \\ 3 Institute of Problems of Microelectronics Technology, \\ 142432 Chernogolovka, Moscow, Russia \\ ${ }^{4}$ CRC Gray Laboratory, Northwood, HA6 2JR, UK
}

\begin{abstract}
In general, the diffraction efficiency of gratings is limited by the constraints imposed by the type of geometry used to scan the photon energy. In the simplest example, the spherical grating monochromator (SGM), the deviation angle, the grating groove width and depth and the groove density are ail constrained by considerations of the maximum photon energy and the tuning range for individual gratings. We have examined the case in which these parameters are unconstrained, resulting in predictions of the ultimate performance of lamellar type gratings for groove densities from 300 to 2400 $1 / \mathrm{mm}$ for gold and nickel coatings. The differential method of Neviere et al [1] was used for modeling the behavior of the gratings and justification is presented for this by rigorous comparison with measurements. The implications of these results for future monochromators based on a variable included angle geometry are discussed.
\end{abstract}

\title{
1. Introduction
}

The design of soft $x$-ray monochromators is a complex task which involves the balancing of a number of opposing factors. In the case of a simple monochromator such as an SGM $[2,3]$ in which the deviation angle defined by the incident beam and the diffracted beam directions is fixed as a function of photon energy, the selection of this angle is driven by the need to have adequate performance at the highest photon energy in the design range. For soft 
$\mathrm{x}$-ray monochromators with a typical upper energy limit of over $1 \mathrm{keV}$, this usually results in the selection of a deviation angle around $174^{\circ}$.

The selection of the line density is then determined for the highest energy grating by consideration of the requirement for a certain minimum energy resolution. In a rigorous optimization, the throughput would be calculated for a defined resolution as a function of line density for a particular photon energy, or a set of photon energies. This optimization depends crucially on the filling of the entrance slit. If the slit is overfilled as is typical for grating monochromators operating on bending magnet second generation sources, there is an advantage in increasing the line density and therefore increasing the slit size required for a particular defined resolution. A balance is reached when further increase of the line density causes a decrease in the product of the fractional slit acceptance and the grating efficiency.

The selection of the highest line density on this basis then automatically determines the line densities for a set number of gratings required to cover the design photon energy range. This results from the minimum photon energy, imposed for a particular line density in a fixed deviation angle geometry. At this minimum energy, or horizon energy, in positive diffraction orders the incident ray is parallel to the grating surface and in negative order diffraction, the diffracted ray is propagated parallel to the surface. It has become conventional to design monochromators with entrance slits to work in positive order and therefore near the horizon energy, the slit size limited resolving power is tending to infinity whereas the dispersive aperture is tending to zero. In the negative order case, the dispersive aperture near the horizon energy is twice that at zero order, but the dispersion is tending to infinity. In a fixed deviation angle design therefore, the designer has to use a number of gratings of decreasing line density to cover lower photon energies.

Having selected the fixed deviation angle, the maximum photon energy and required resolving power set the value of the maximum line density, and the lower line densities are set by the total number of gratings to be used and the minimum photon energy required. The groove depth and width are then selected for each grating either by maximizing the peak diffraction efficiency at a certain energy or by maximizing the integrated intensity within the design range of the grating. For VUV monochromators, a choice also has to be made as to whether to maximize the diffracted intensity in first order or to maximize the ratio of the intensity of first order to second or higher order light. For a groove width to period ratio of 
0.5 giving a symmeiric groove width and land width, complete suppression of even order radiation can be achieved in normal incidence geometry. Even with significantly off normal geometry such as in the Seya Namioka monochromator with a deviation angle of $70^{\circ}[4]$, extremely good even order suppression can be achieved. At grazing incidence however, the effects of groove shadowing reduce the effect of this even order rejection to a large extent and it is usual simply to maximize the first order diffracted intensity. This typically results in a groove width to period ratio of around 0.65 . Selection of the groove depth is then arranged to produce the first interference maximum of the grating at the center of the design energy range for the grating [5].

The purpose of this paper is to examine the case of a completely unconstrained optimization in which we allow free selection of the deviation angle, the groove width and groove depth for a range of photon energies from $100 \mathrm{eV}$ to $2000 \mathrm{eV}$. These calculations have been performed for a wide range of line densities, from 300 to $24001 / \mathrm{mm}$ of which some examples are given here, and for both gold and nickel reflectors. For each line density therefore we predict the conditions for maximum efficiency as a function of photon energy, as well as the efficiency itself. In addition, we consider the practical realization of a design in which we could have the selection of these unconstrained parameters.

\section{Evaluation of the theoretical model by comparison with experiment}

The model used for prediction of the diffraction efficiency of lamellar gratings was that due to Neviere et al [1]. Many other models exist such as the scalar model of Franks et al [5], but the model of Neviere is the only approach that attempts to rigorously solve the problem as an electromagnetic periodic boundary problem. There are a whole class of such solutions and an introduction to the subject is given by Petit [6]. In addition, a comprehensive description of the differential method is given by Valdes and McKinney [7]. Figure 1 shows the definition of the angles of incidence and diffraction and the signs of the orders. It also gives the three regions for which solutions can be found, E1 above the grating surface, E2 within the lands and grooves and E3, in the bulk of the material beneath the grating structure. The grating shape has only to be periodic, and as it is represented as a Fourier series, solutions can be found for any of the commonly used shapes, sinusoidal, blazed or as considered here, lamellar

or rectangular. As the method gives a complete solution of the periodic boundary problem, it 
automatically deals with penetration through the lands and associated phase shifting. The energy dependent complex refractive index of the surface was derived from the atomic scattering factors tabulated by Henke et al [8].

As we wished to apply the model to a wide range of problems, we have attempted to verify the model by testing it against measurements over as wide an angular and energy range as possible using several high quality grating substrates. The measurements were conducted using a carbon $\mathrm{K}$ line source $(278 \mathrm{eV})$, as well as using monochromatized synchrotron radiation for measurements up to $800 \mathrm{eV}$. The apparatus used for these diffraction efficiency measurements is shown in Fig. 2. It consists of a standard vacuum diffractometer with coaxial rotation axes for the sample and the detector as well as an $x-y$ table for movement of the grating along and perpendicular to the rotation axis. A GaAsP photodiode was used for detecting diffracted synchrotron light [9], and for the lower intensity laboratory line source, a pulse counting channeltron was used [10]. The rotations were externally actuated from stepping motor driven high precision rotary tables [11] with the rotation being transmitted through into the vacuum system via a coaxial differentially pumped rotary feedthrough. The minimum rotation increment was $0.0005^{\circ}$ and the absolute accuracy over the range of motions used here was $0.005^{\circ}$. The $x-y$ stage had a total motion for each axis of $50 \mathrm{~mm}$ and allowed selected areas of the gratings to be measured. It was therefore possible to measure the diffraction efficiency as a function of position as well as to infer the local groove depth. The collimation was set by an aperture $\mathrm{S} 1$ which was adjustable in position, and by a combination of a fixed horizontally defining slit, S3, and an externally adjustable vertically defining slit, S2. Initial alignment of the slits, apertures and rotation axes was accomplished using an optical level to an accuracy of $0.1 \mathrm{~mm}$.

Figure 3 shows a typical detector scan from a $1200 \mathrm{l} / \mathrm{mm}$ grating at an incidence angle of $3^{\circ}$ degrees for a photon energy of $278 \mathrm{eV}$. The figure shows a large zero order peak at an angle of grazing diffraction of $3^{\circ}$, as well as the first and second positive order peaks at higher angles. It is clear that the positive order peaks are wider than the zero order peak and this effect is due to the asymmetric nature of the diffracting geometry. Positive orders will be expanded and negative orders will be compressed, the effect increasing with order number. It is necessary therefore to use an angular resolution smaller than the narrowest and lowest order feature in the distribution to be recorded. The true efficiencies are then extracted by integrating each peak and taking the ratio to the incident beam. This latter step was done 
periodically by translating the grating out of the beam using the $x-y$ stage, measuring across the incident beam and integrating the distribution. This whole process was repeated for each angle of incidence to be measured.

Figure 4 shows the diffraction efficiency as a function of incidence angle for a $12001 / \mathrm{mm}$ gold coated grating at a photon energy of $278 \mathrm{eV}$ for orders of diffraction from -2 to +2 . It can be seen that the orders show a marked oscillatory behavior as expected and very gcod repeatability between the two data sets shown for each order. The dotted line shows the best fit for the model over the + and - 1st orders, using the groove depth and width as fitting parameters. The groove depth inferred from this measurement was $127 \AA$ compared to the specification of $100 \AA$. It is apparent that there is an excellent fit for the zeroth and first orders over their whole, angular range. The peak positions and intensities are well reproduced by the model, the only discrepancy being an increase in the diffraction efficiency in the region of the interference minima in zeroth and first positive order given by experiment in comparison to theory. This can be explained by approximately a $10 \%$ variation in groove depth over the measured region. A small change in gradient can be seen in the region of $6^{\circ}$ for the +1 order and $8.6^{\circ}$ for the -1 order both in the theory and experiment. These angles correspond to a position where a higher order is propagated parallel to the surface and is conventionally known as a Woods anomaly. At this angle, part of the energy diffracted into the beam traveling parallel to the surface is transferred to other orders. It is reassuring that such a subtle effect is faithfully reproduced by our theoretical model. The measured second order intensities are generally a factor of two higher than the predictions of the model. Measurements of several gratings showed that whilst the first order diffraction efficiency was very similar to the data presented here, there were significant differences in the amplitude and form of the second order measurements. This indicates that the difference is an experimental effect, probably caused by imperfections in the groove shape.

The data can also be plotted as a function of the deviation angle instead of the incidence angle, and this is shown for the first and second orders in Fig. 5. The solid symbols represent the positive orders and the open symbols the negative orders. It is clear that there is a complete overlap of the opposite orders and provides direct evidence of the reciprocity theorem of Maystre and McPhedran [12]. It also implies that a choice between negative and positive order operation in a monochromator can simply be based on geometrical grounds. For example, for a soft x-ray SGM, from considerations of the optimum focusing, there is 
approximately a two to one ratio between the length of the entrance and exit arms lengths in negative order and the opposite ratio in positive order. For an entrance slitless monochromator used on a high energy storage ring, the radiation shielding usually prevents positioning of optical elements closer than $15 \mathrm{~m}$ from the tangent point. Whilst a negative order geometry would give a reasonable exit arm length of $7.5 \mathrm{~m}$, the positive order solution would give an unreasonably large distance $[13,14]$.

Many gratings have been tested, using both nickel and gold coatings, over line densities from 600 to $1800 \mathrm{l} / \mathrm{mm}$ and photon energies from 278 to $800 \mathrm{eV}$. In all cases where we have verified the uniformity and quality of the surface using contact profilometry or laser diffraction, we have obtained excellent agreement with the model of Neviere et al. In the only cases where we have seen a discrepancy between measurement and theory, this has been found to be due to faulty manufacture of the grating.

\section{Prediction of optimized performance.}

Having verified the accuracy of the model, we have used it to predict the optimized performance of lamellar diffraction gratings. After selecting a particular groove density, photon energy and coating material, we first pick a deviation angle and then search over a region of the $2 \mathrm{~d}$ space defined by the groove width and depth in which we expect to find the first interference maximum. A graph showing such a function is shown in Fig. 6 for a 1200 $1 / \mathrm{mm}$ grating at $278 \mathrm{eV}$ photon energy and for an angle of grazing incidence of 3.2 degrees. It can be seen that there is a broad maximum in the distribution both as a function of groove depth and groove width. The program then stores the maximum value of the diffraction efficiency, the position of the maximum in groove depth and width and the deviation angle. The deviation angle is then incremented and the search process repeated until a principal maximum is found. All the values are then stored and the photon energy is then incremented. This process is repeated for the whole required photon energy range, in this case 100 to 2000 $\mathrm{eV}$.

The data resulting from such an optimization is shown in Fig. 7 and 8 respectively for gold and nickel coatings, for line densities of 300 and $9001 / \mathrm{mm}$. These are examples of data calculated for gratings with line densities from 300 to $24001 / \mathrm{mm}$ in increments of $300 \mathrm{l} / \mathrm{mm}$ [15]. The top graph of each panel gives the maximum diffraction efficiency that can be obtained for that 
particular line density as a function of photon energy, and the lower panels give the deviation angle, the groove depth and groove width that must be used to achieve the maximum. In addition, the center panel that shows the deviation angle as a function of photon energy also gives the deviation angles that would have to be used to achieve $75 \%, 50 \%$ and $25 \%$ of the maximum, assuming the groove depths and widths shown in the lower panel, and therefore represent an error bar or detuning function.

It can be seen that the diffraction efficiency curves shown in Fig. 7 for nickel exhibit very high diffraction efficiency up to around $800 \mathrm{eV}$, followed by a sharp decrease. This decrease is due to the onset of the nickel L2,3 absorption edges. It is also apparent that as the line density increases, the diffraction efficiency decreases and that the deviation angles necessary to achieve the maximum efficiency decrease with increasing line density. The photon energy at which the maximum efficiency occurs also tends to decrease with increasing line density. All these trends are confirmed from our full data sets for gratings from 300 to $24001 / \mathrm{mm}$. The results for gold are shown in Fig. 8 and show similar effects to those previously described for nickel. The main differences are a lower average diffraction efficiency for the region lower than the nickel L2,3 edges and a sudden increase in efficiency between 100 and $200 \mathrm{eV}$. This latter effect is probably related to the extra oscillator strength from the presence of the $\mathrm{N}_{6,7}$ edges at around $88 \mathrm{eV}$.

These graphs therefore provide the optical designer with a method of easily predicting the optimum performance of a grating. This can be useful in designing a monochromator in which the photon energy range is limited, such as 'water window' $\mathrm{x}$-ray microscopy, as well as allowing a proper comparison of a constrained solution to the optimum. Such a constrained solution might be to first use these graphs to fix the optimum conditions at a particular photon energy, then to fix the variables of deviation angle and the groove parameters and calculate the efficiency as a function of photon energy. This would then allow comparison of an optimized SGM for example against a fully unconstrained design.

\section{Practical realization of an optimized geometry.}

The optimization graphs strongly suggest that to achieve a high diffraction efficiency over a wide energy range, a variable deviation angle geometry has to be used. The most widely 
used example of this geometry is that of the SX700 due to Petersen [16], although variable angle versions of other monochromators such as the SGM also exist [14, 17]. A later adaptation of this monochromator is shown in Fig. 9. The concept is the same with a rotatable plane pre-mirror and rotatable plane grating arranged so that there can be any chcice of the angle of incidence $(\alpha)$ and the angle of diffraction $(\beta)$ at the grating. The focusing mirror in the original SX700 was an ellipsoid and therefore provided focusing in the tangential and sagittal directions. In the adaptation shown in Fig. 9, this ellipsoidal mirror has been replaced by a spherical mirror working at low demagnification and was first described by Padmore [14] and later by Reininger and Saile [18]. This therefore provides focusing only in the tangential direction (at grazing incidence), the sagittal focusing being performed by an ellipsoidal mirror after the exit slits. In this case it is possible to allow the light to diverge in the sagittal direction through the monochromator until reaching the ellipsoidal refocusing mirror after the exit slits, as the light from the undulator source is highly collimated. In the case of the application of this type of monochromator on a bending magnet source, a solution would be to use a horizontally deflecting tangential cylindrical mirror as the first element in the beamline.

The most common method of interchanging gratings is to mount them on a slideway that is directly connected to the rotation axis, but allows lateral interchange of the gratings using a linear actuator. The aim is therefore to have a grating with a variable groove depth and width along the direction of the grating grooves so that lateral motion of the grating can bring the region with the appropriate groove parameters into the beam. This is only possible if the beam dimension along the grooves is small compared to the total dimension of the grating in the same direction. This will be the case for beamlines using undulator sources on third generation storage rings in the soft $x$-ray region. As an example, for a $5 \mathrm{~m}$ long undulator operating at a wavelength of $44 \AA$ on the Advanced Light Source, the angular width of the single electron radiation pattern convoluted with the divergence of the electron beam in the horizontal direction is approximately $42 \mu \mathrm{rad}(1 \mathrm{sigma})$. The electron beam width in the horizontal direction is $0.33 \mathrm{~mm}$ ( 1 sigma) at the center of the undulator, and so using a typical distance from the source to the grating of $15 \mathrm{~m}$, the combined full width at half maximum of the photon beam in the horizontal direction at the grating is $1.7 \mathrm{~mm}$. In practice, more than the central cone is usually accepted but even so, the beam width is far smaller than the width of a typical grating of around $50 \mathrm{~mm}$. The grooves are produced in the substrate by ion milling through a mask of photoresist produced by a holographic lithography process. To alter 
the groove depth as a function of position along the groove direction, the grating would be initially covered with a baffle during the ion etching process which would then be drawn back to expose the grating at a rate calculated to yield the desired variation in depth with position. This would appear to be an easily accomplished process and could yield significant benefit. In practical use, the gratings would be translated to a position that gave a groove depth appropriate for the center of the range to be measured, and then fixed. Most experiments in the soft $x$-ray energy range usually require only a relatively narrow scanning range, and the use of a fixed set of groove parameters over such a range would not result in a significant deviation from optimum performance.

A plane grating produces a focusing action that is wavelength dependent and can be described by [19],

$$
r=r \frac{\cos ^{2} \beta}{\cos ^{2} \alpha}=r C^{2}
$$

where $r^{\prime}$ is the virtual image position and $r$ is the real object position. As the focusing mirror has a fixed radius and a fixed angle of incidence, the use of a fixed exit slit position constrains the ratio of $\cos \beta / \cos \alpha$ to be fixed (and equal to $C$ ) if the monochromator is to be in focus.

In section 3, we described the optimization of diffraction efficiency in the case of no constraints, allowing free choice of the groove parameters and the deviation angle. As can be seen from above, a completely free choice of the deviation angle will result in a photon energy dependent virtual image position and hence to defocusing by a fixed mirror, fixed exit slit design. The question arises therefore as to the effect of constraining the deviation angle to follow the condition for a fixed focus, whilst still having the freedom to choose the appropriate values of the groove width and depth. Fig. 10 shows a comparison between the unconstrained optimization (dotted line) and the optimization constrained by having a constant virtual image distance (solid line). We have the freedom to choose any value of the constant $C=\cos \beta / \cos \alpha$ and therefore we searched over all values of $C$ to find the optimum value. For the case of a $1200 \mathrm{l} / \mathrm{mm}$ gold coated grating working in +1 order, the optimum value of $C$ is 2.166. It can be seen that throughout the entire energy range of 100 to $2000 \mathrm{eV}$, the variation in deviation angle for the unconstrained and the constrained case are very similar, leading to near identical diffraction efficiency curves. With the optimum choice of $\mathrm{C}$ therefore, we can 
have a design that stays in-focus and on blaze throughout a wide energy range, if we have the freedom to choose the appropriate groove parameters by lateral translation of the grating.

A further question is, if we have constrained the deviation angle by making $\cos \beta / \cos \alpha$ constant, and we constrain the groove parameters of groove width and depth to be constant, then if we have the freedom to choose the optimum value of $C$, how does the solution compare to the unconstrained case. The results of this for a $1200 \mathrm{l} / \mathrm{mm}$ gold coated grating are shown in Fig. 11. The results here are shown for a value of $C=2.094$ which gives optimum performance in the 600 to $900 \mathrm{eV}$ range. Changing the value of $\mathrm{C}$ changes the energy position of this optimum. This value of $\mathrm{C}$ also gives the highest value of diffraction efficiency integrated over the whole energy range from 100 to $2000 \mathrm{eV}$. Three cases are shown, (1) with unconstrained parameters (long dash), (2) with a free choice of the deviation angle but with a fixed groove width of $5300 \AA$ and a fixed groove depth of $70 \AA$ (short dash), and (3) with the same fixed groove parameters but with the deviation angle constrained by $\mathrm{C}=2.094$ (solid). It can be seen that the fully constrained case compares well with the unconstrained case from 500 to $2000 \mathrm{eV}$, but at low photon energies the deviation between the two is very marked. Even using an unconstrained choice of the deviation angle with fixed groove parameters only produces a small improvement. The number of additional gratings necessary to optimally cover this lower energy region will be the subject of future study.

\section{Optimization of throughput}

The previous sections considered the case where diffraction efficiency is to be optimized. In practice, resolution is always of concern, even in cases where only modest resolution is required such as diffraction limited $x$-ray microscopy. In this case, the optimization graphs could be used to choose the lowest line density for which the combination of the line density and the deviation angle give the desired source size limited resolution at the optimization wavelength, $\lambda$. As we have seen previously, a reduction in line density always leads to an increase in efficiency. The resolution can be found from the grating equation,

$$
d \lambda=\frac{1}{N m} \frac{s}{r} \cos \alpha
$$


where $\mathrm{N}$ is the line density, $\mathrm{m}$ is the order of diffraction, $\mathrm{s}$ is the source size, $\mathrm{r}$ is the source distance, and $\alpha$ is the angle of incidence which can be related to the deviation angle $2 \theta$ by,

$$
\alpha=\sin ^{-1}\left[\frac{N m \lambda}{2 \cos \theta}\right]+\theta
$$

A second type of optimization would be used for the case where the entrance slit of a monochromator is always expected to be overfilled, but the grating is always underfilled. This is a common situation for high resolution monochromators working on second generation bending magnet light sources. It can be seen from the above equation for the source size limited resolution that the slit size, and hence flux in this case, is proportional to $\mathrm{N} / \cos \alpha$ for a fixed resolution. This dependence on the angle of incidence means that the optimization graphs cannot be used directly. In searching for the optimum efficiency by varying the deviation angle, the efficiency should be weighted at each point by $N / \cos \alpha$. This type of optimization will be the subject of future study.

A third type of optimization would be for the case of a system in which the entrance slit and grating are always overfilled. This is the usual case in a spectrograph used to energy resolve fluorescence radiation. The angular aperture of the grating is simply equal to $(\mathrm{L} / \mathrm{r}) \cos \alpha$, where $L$ is the grating length and $r$ is the slit to grating distance. Combining this with the expression for the slit size given above simply gives the phase space acceptance at fixed resolution as proportional to $\mathrm{N}$. The optimization graphs can then be simply used by maximizing the product of the efficiency and the grating density for the desired photon energy.

\section{Acknowledgment}

This work is supported by the Science and Engineering Research Council, UK, and by the Director, Office of Energy Research, Office of Basic Energy Sciences, Materials Sciences Division of the U.S. Department of Energy under contract Number DE-AC03-76SF00098. 


\section{References}

1. M.Neviere, P. Vincent and R. Petit, Nouv. Rev. Opt. 5 , 65 (1974)

2. C. T. Chen and F. Sette, Rev. Sci. Instrum. 60, 1616 (1989)

3. H. Hogrefe, M.R. Howells and E. Hoyer, SPIE $\underline{733}$, 174 (1986)

4.. M. Seya, Sci. Light 2,8 (1952)

T. Namioka, J. Opt. Soc. Am. 49951 (1959), and

T. Namioka, Sci. Light 3 , 15 (1954)

5. A. Franks, K. Lindsey, J. M. Bennett, R. J. Speer, D. Turner and D. J. Hunt, Philos. Trans. Roy. Soc. London, A277, 503 (1975)

6. R. Petit, Ed., Electromagnetic Theory of Gratings, Topics in Current Physics, vol. 22, (Springer-Verlag, Berlin 1980)

7. V.S. Valdes, and W. R. McKinney, this volume

8. B. L. Henke, P. Lee, T. J. Tanaka, R. L. Shimabukuro, and B. K. Fujikawa, At. Data Nucl. Data Tables 27 , 1 (1982)

9. GaAsP/Au photo diodes. Hammamatsu type G1127/04 and G2119/01

10. Channeltron, Gallileo type 4800

11. Rotary table. Microcontrol type RT 200

12. D. Maystre and R. C. McPhedran, Opt. Comm. 12, 164 (1974)

13. H. A. Padmore, SPIE $\underline{733}, 253$ (1986)

14. H. A. Padmore, Rev. Sci. Instrum. 60 , 1608 (1989) 
15. H. A. Padmore, V. Martynov and K. Hollis (to be published)

16. H. Petersen, Opt. Commun. $\underline{40} 402$ (1982)

17. W. B. Peatman and F. Senf, Proc. 10th Int. Conf on Vacuum Ultraviolet Radiation Physics, VUV10, Paris, July 1992

18. R. Reininger and V. Saile, Nucl. Instr. Meth. A307, 135 (1991)

19. M. V. R. K. Murty, J. Opt. Soc. Am. $\underline{52} 768$ (1962) 


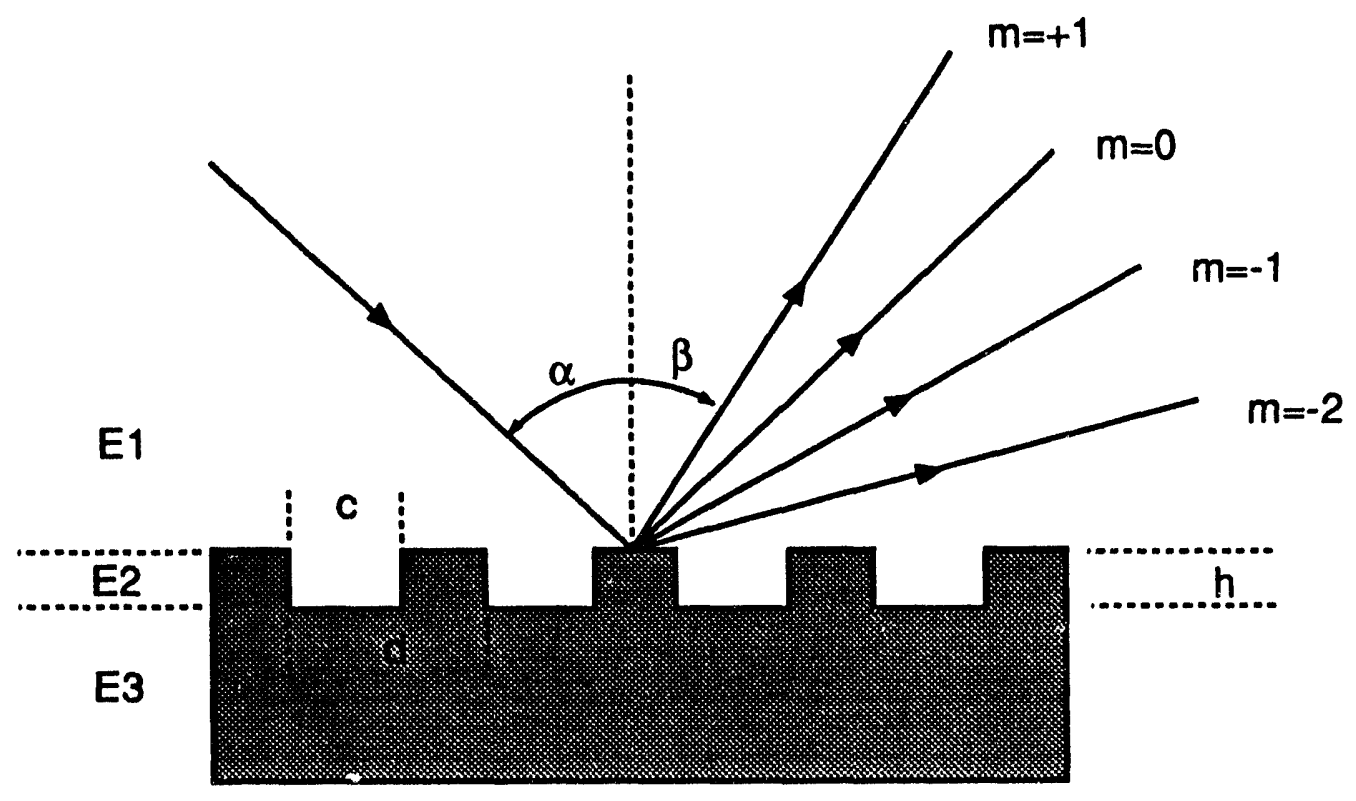

Fig. 1

Definition of the sign of the diffraction orders, the grating parameters and the three regions of solution for a lamellar grating, E1 above the grooves, E2 in the grooves and lands, and E3 in the substrate. 

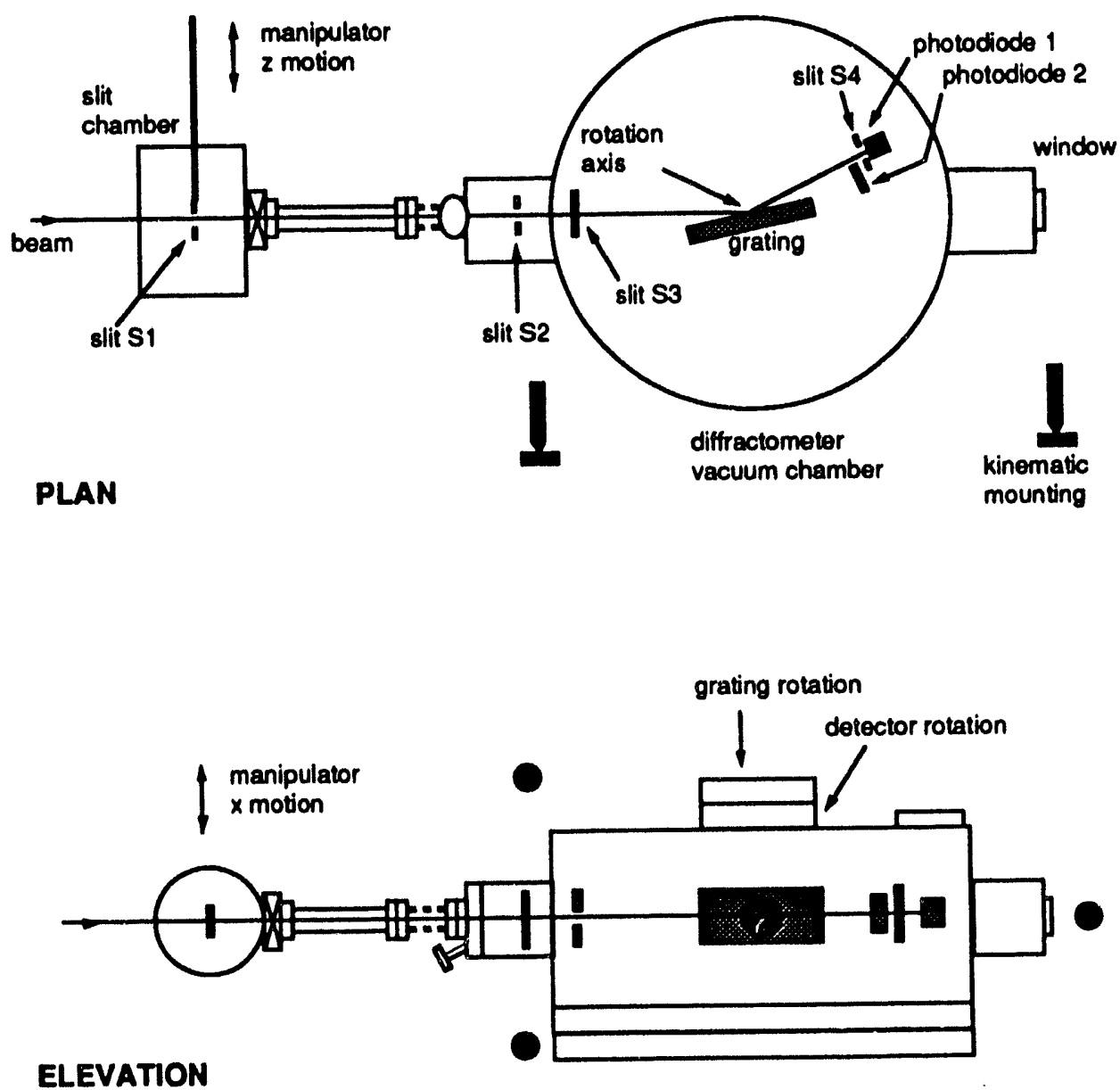

Fig. 2

Schematic shows the arrangement of the two axis vacuum diffractometer. 


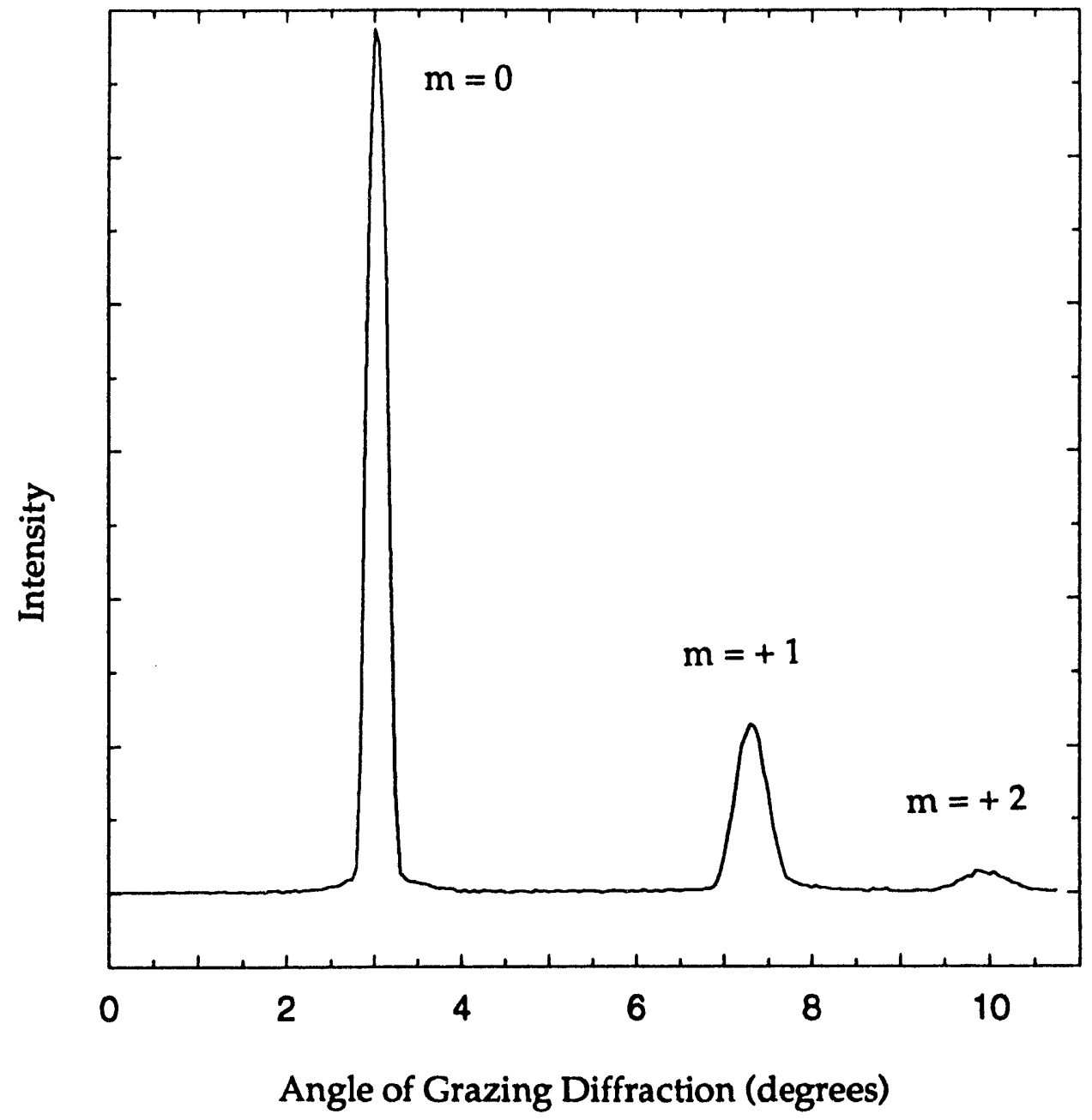

Fig. 3

Diffracted intensity as a function of the angle of grazing diffraction for a $1200 \mathrm{l} / \mathrm{mm}$ grating at a photon energy of $278 \mathrm{eV}$. 


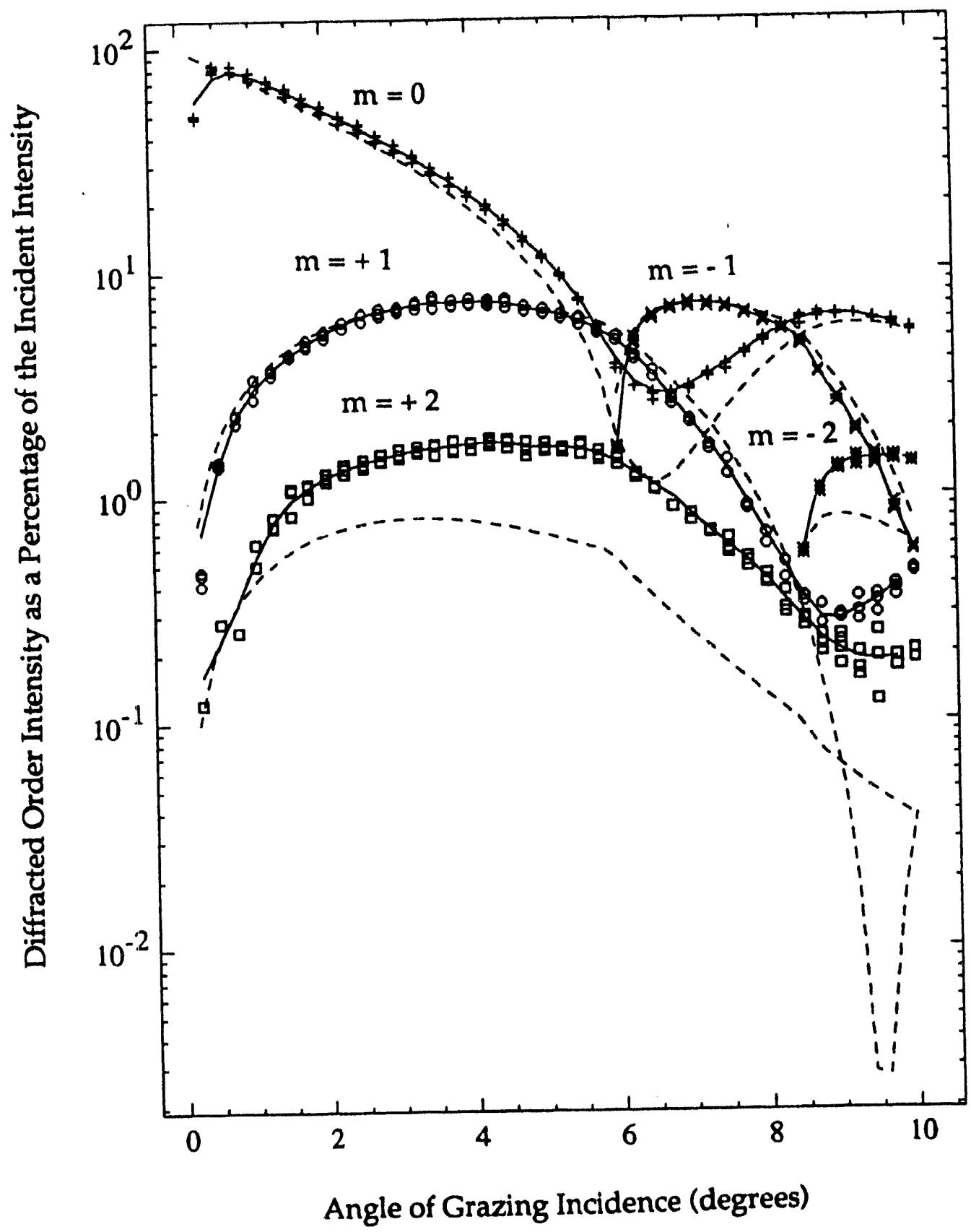

Fig. 4

Absolute diffraction efficiency for the -2 to +2 orders as a function of the angle of grazing incidence for a $1200 \mathrm{l} / \mathrm{mm}$ grating at a photon energy of $278 \mathrm{eV}$. The symbols denote measured data and the dashed lines indicate the theoretical model fitted to the $m=+1$ data with a groove depth of $127 \AA$. 


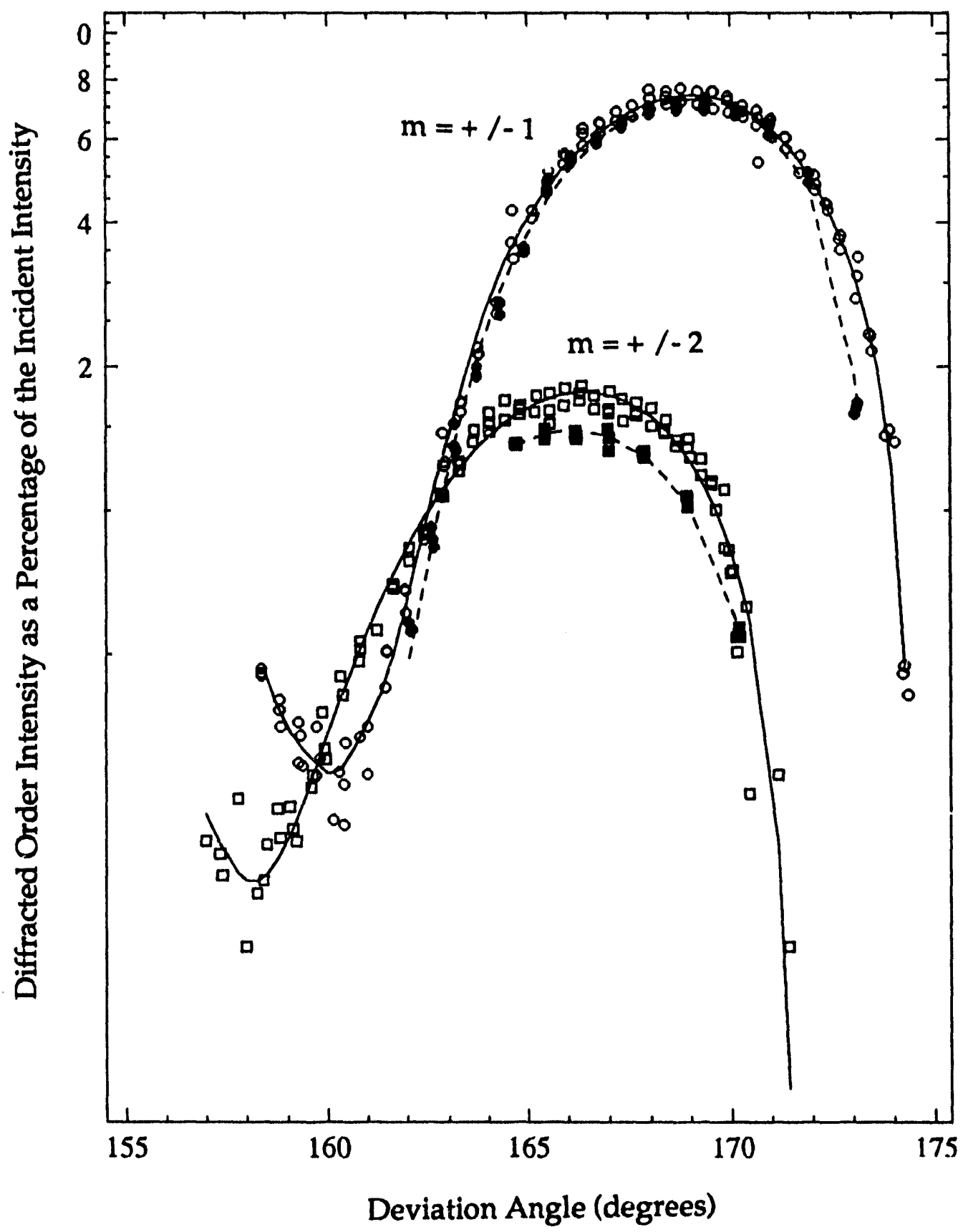

Fig. 5

Absolute diffraction efficiency for the $m=+1$ and -1 orders shown in Fig. 4 plotted as a function of the deviation angle, ie. the angle defined by the incident and diffracted beams. 


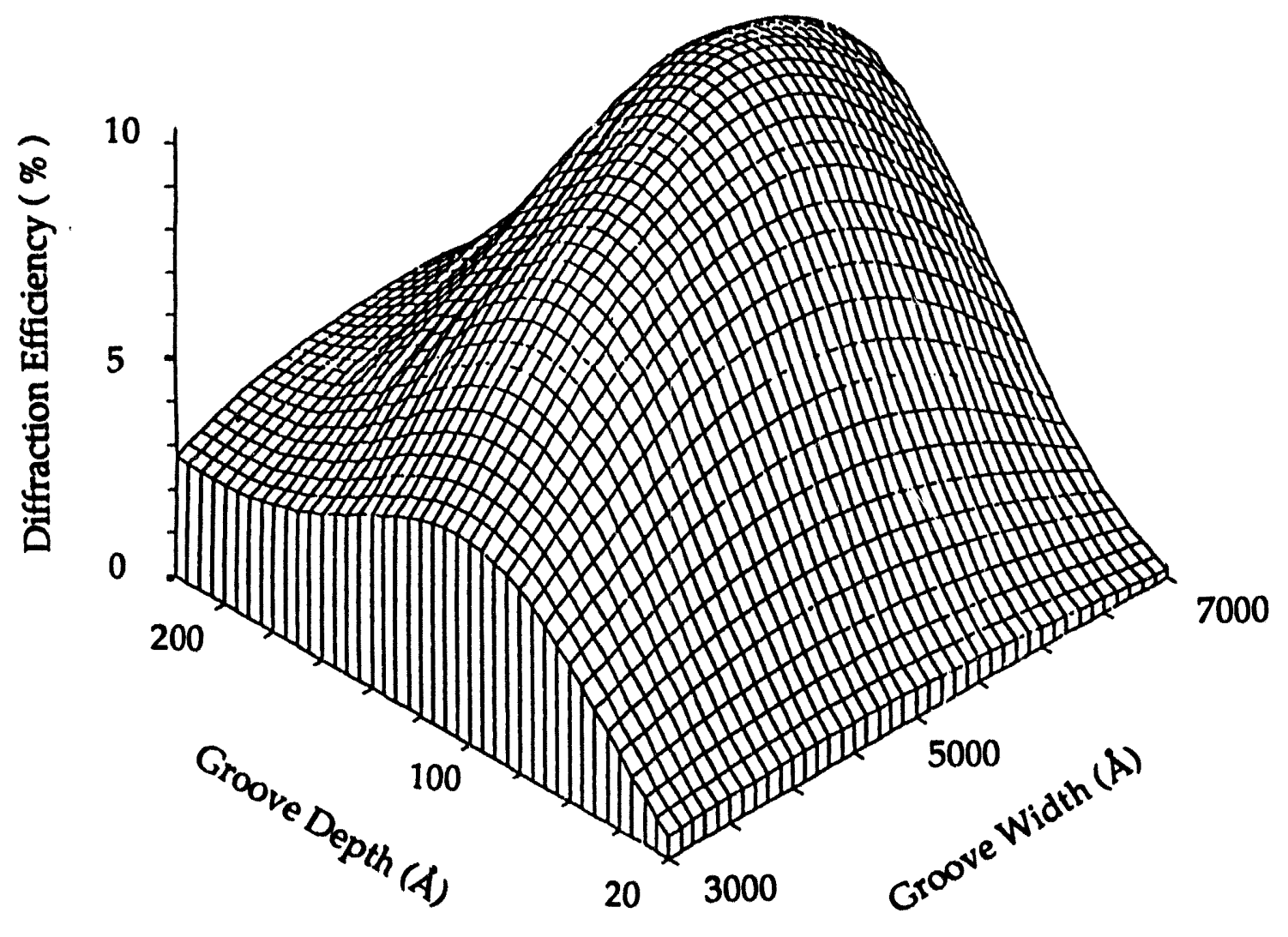

Fig. 6

Theoretical diffraction efficiency as a function of the groove depth and groove width for a 1200 $1 / \mathrm{mm}$ grating at a photon energy of $278 \mathrm{eV}$ and for an angle of grazing incidence of $3.2^{\circ}$. 


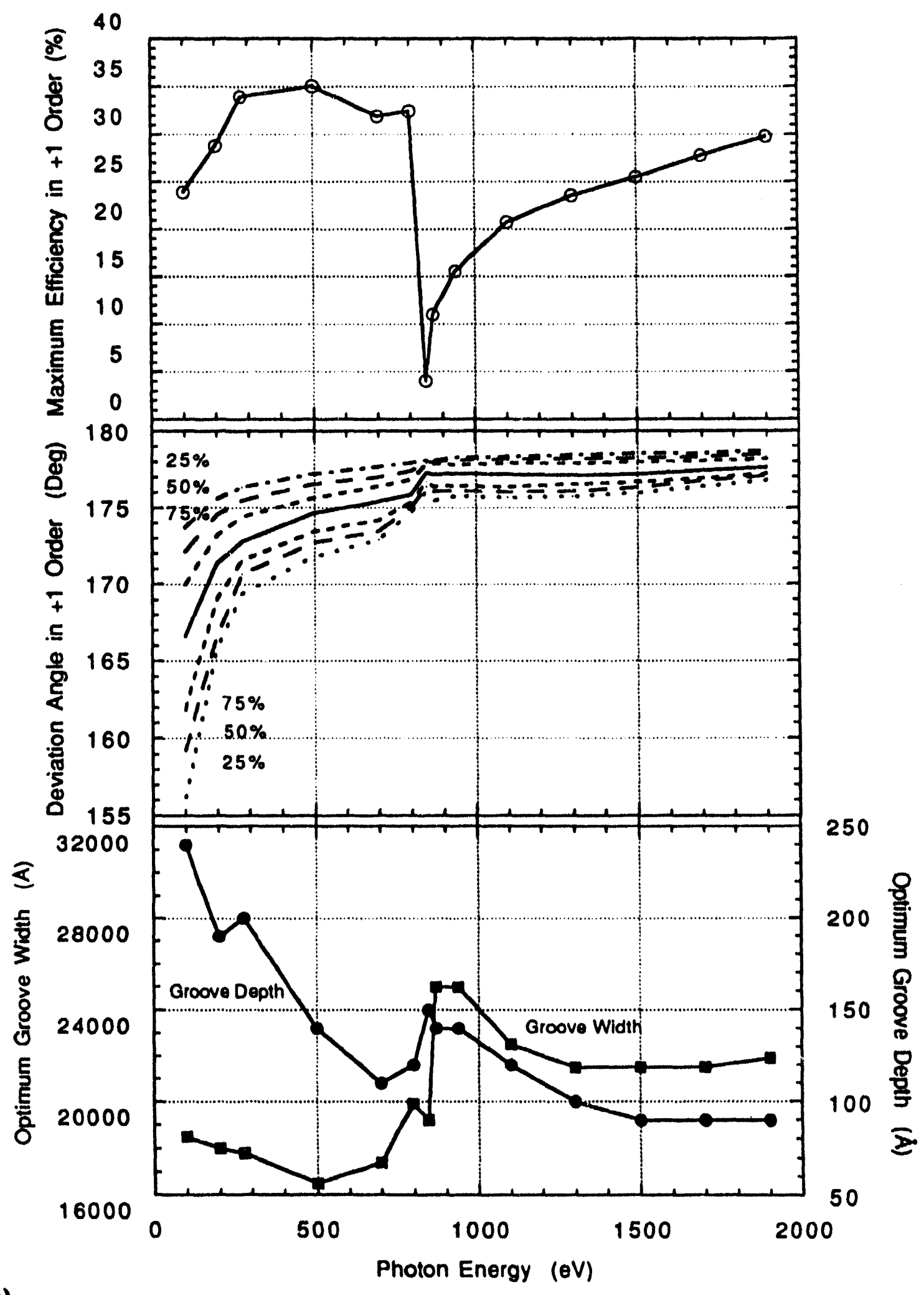

Fig. 7(a)

Theoretical maximum diffraction efficiency for a nickel grating of $3001 / \mathrm{mm}$ as a function of photon energy. The upper panel shows the maximum efficiency, and the middle and lower panels show the conditions necessary to achieve the maximum. In addition the center panel shows the deviation angles required to give $75 \%, 50 \%$ and $25 \%$ of the maximum efficiency with the use of the optimum groove parameters. 


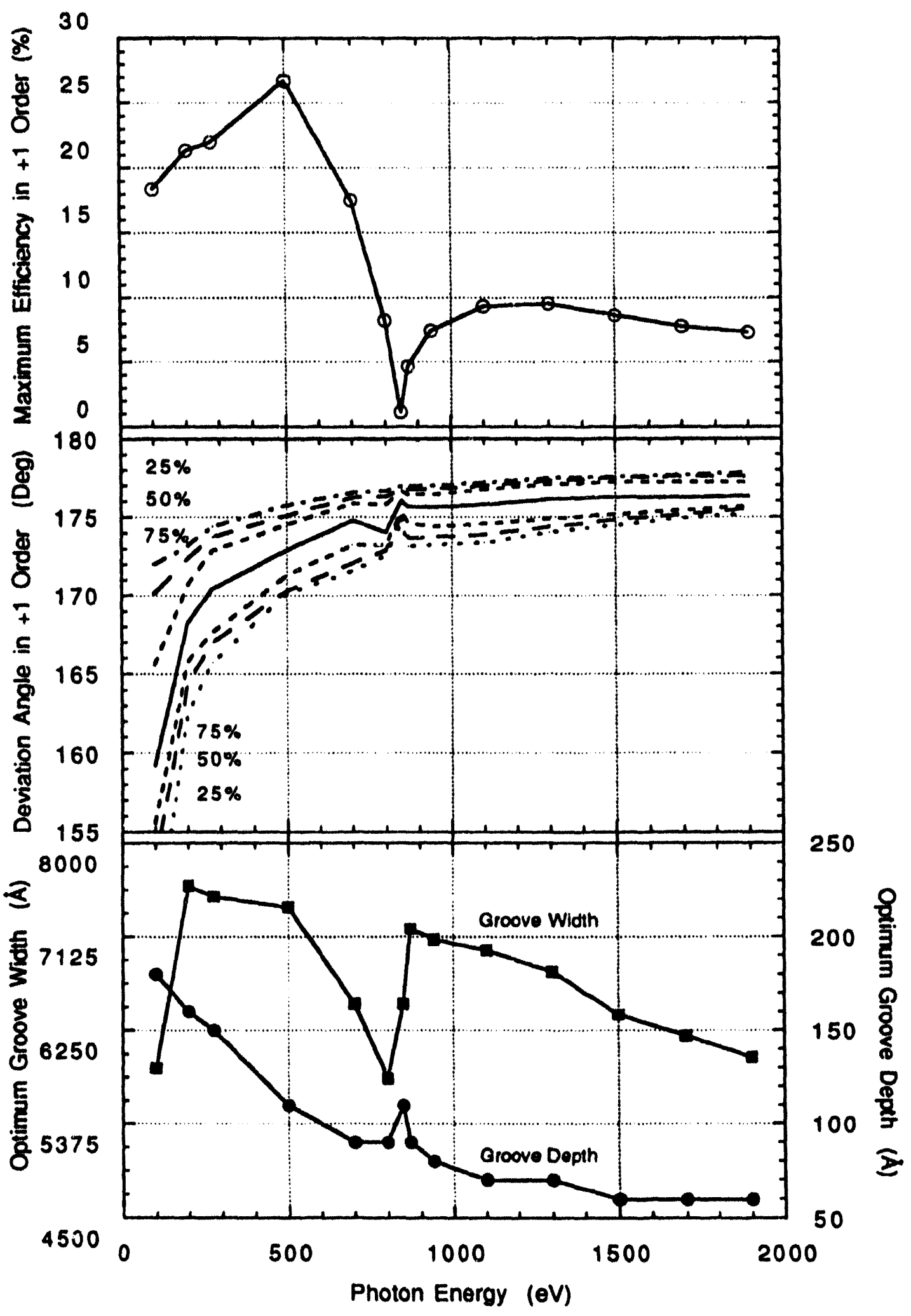

Fig. 7(b)

Theoretical maximum diffraction efficiency for a nickel grating of $9001 / \mathrm{mm}$ as a function of photon energy. The upper panel shows the maximum efficiency, and the middle and lower panels show the conditions necessary to achieve the maximum. In addition the center panel shows the deviation angles required to give $75 \%, 50 \%$ and $25 \%$ of the maximum efficiency with the use of the optimum groove parameters. 


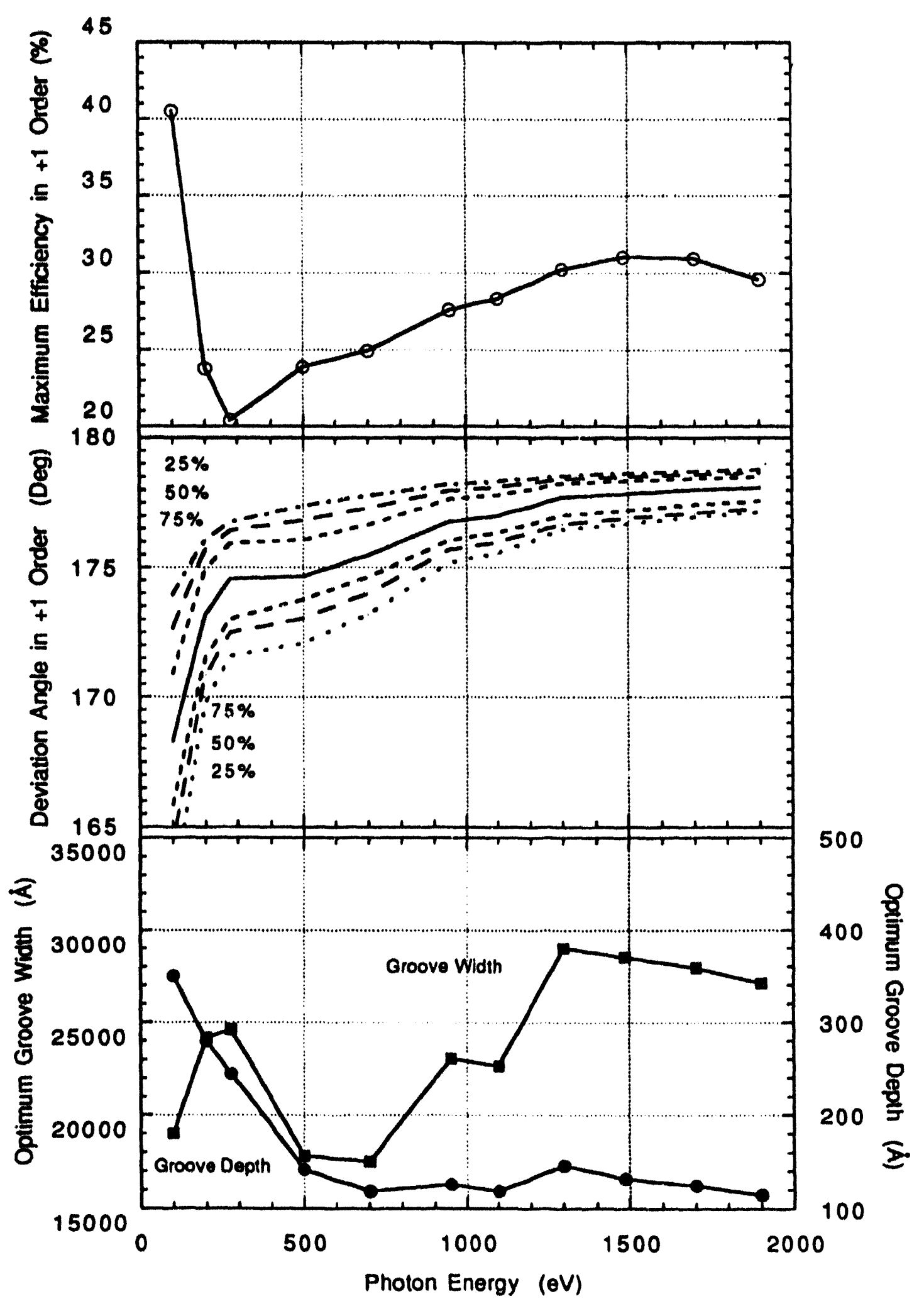

Fig. 8(a)

Theoretical maximum diffraction efficiency for a gold grating of $300 \mathrm{1} / \mathrm{mm}$ as a function of photon energy. The upper panel shows the maximum efficiency, and the middle and lower panels show the conditions necessary to achieve the maximum. In addition the center panel shows the deviation angles required to give $75 \%, 50 \%$ and $25 \%$ of the maximum efficiency with the use of the optimum groove parameters. 


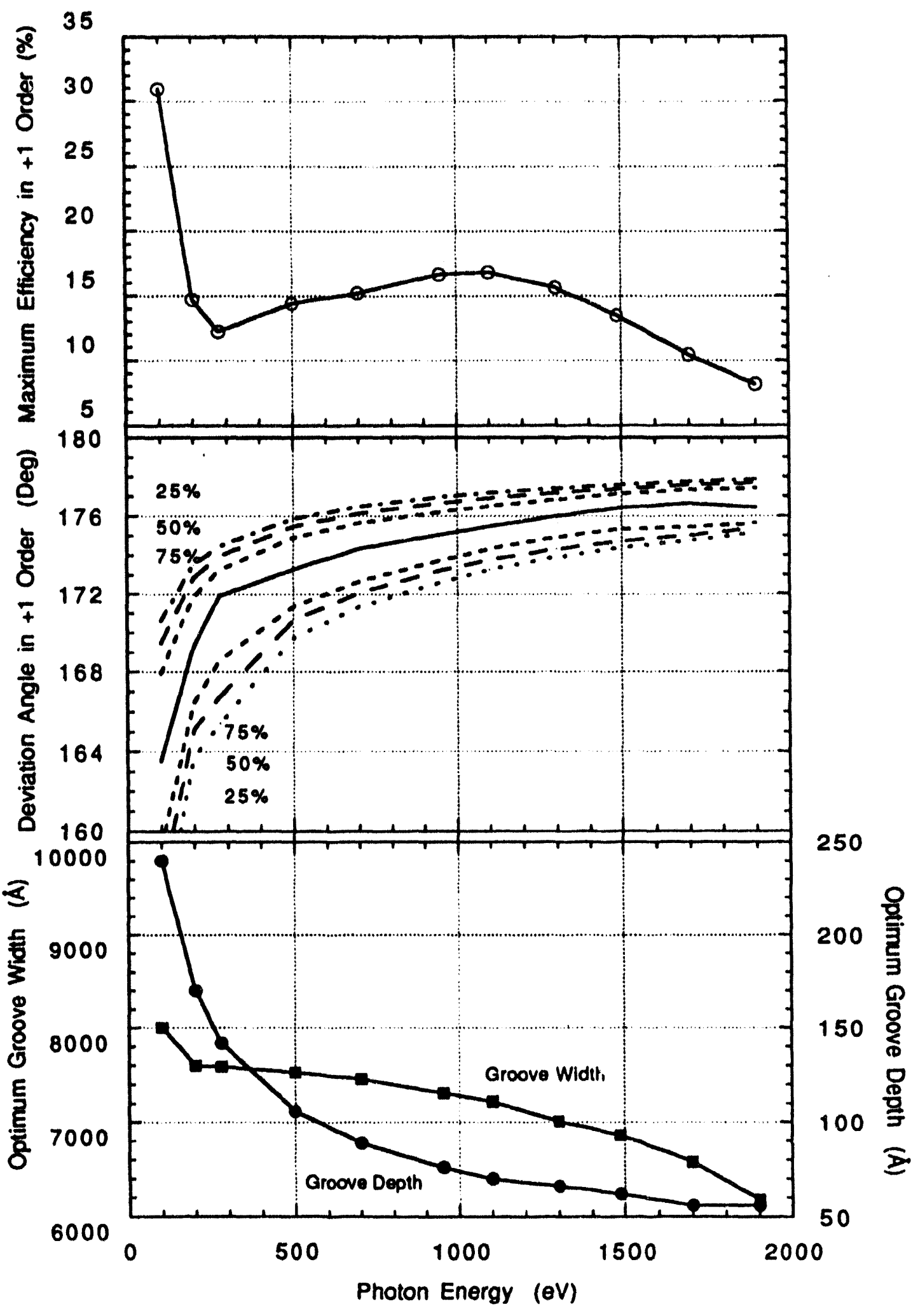

Fig. 8(b)

Theoretical maximum diffraction efficiency for a gold gratings $9001 / \mathrm{mm}$ as a function of photon energy. The upper panel shows the maximum efficiency, and the middle and lower panels show the conditions necessary to achieve the maximum. In addition the center panel shows the deviation angles required to give $75 \%, 50 \%$ and $25 \%$ of the maximum efficiency with the use of the optimum groove parameters. 


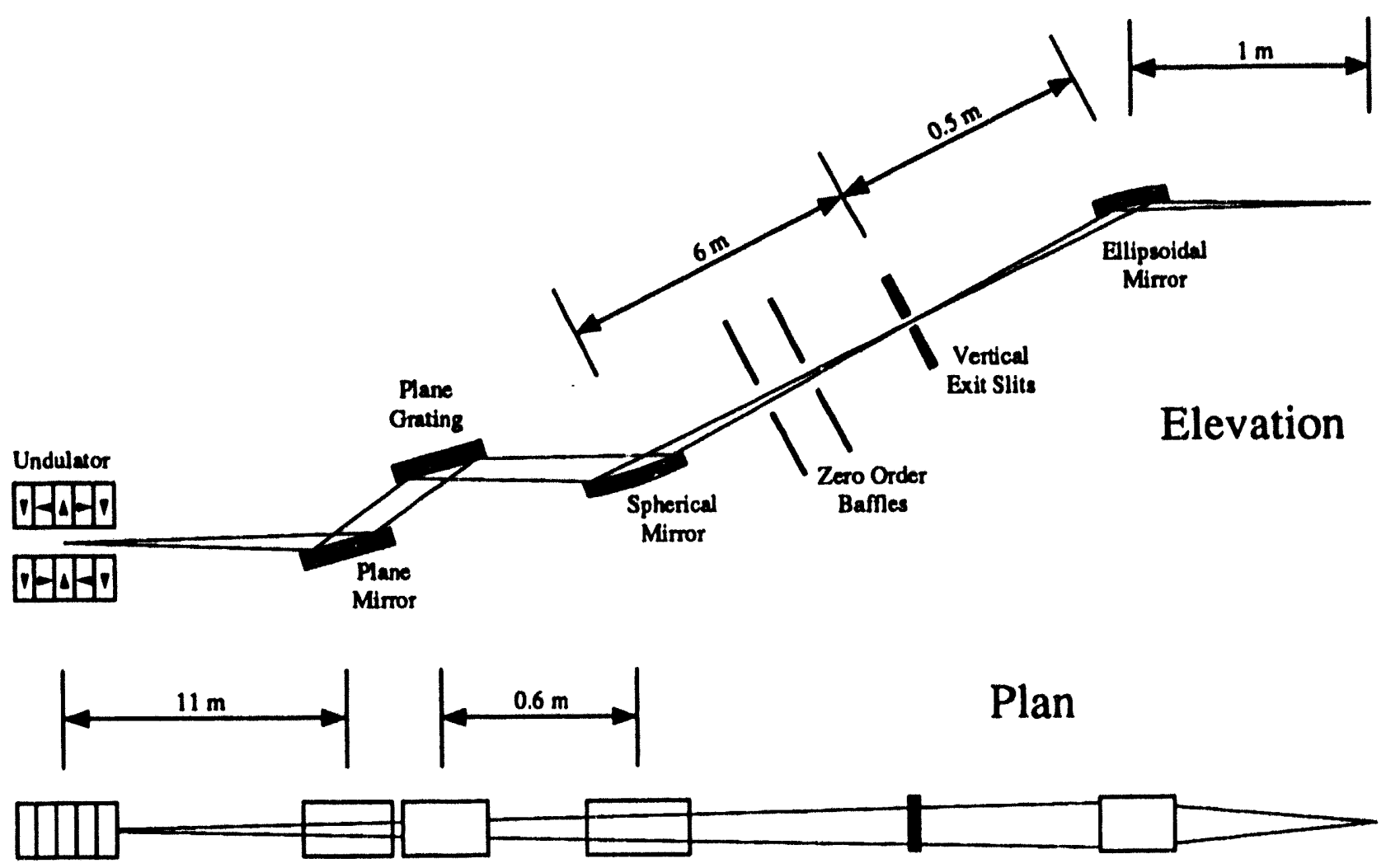

Fig. 9

Schematic arrangement of a variable deviation angle monochromator of the SX700 variety but using a spherical focusing mirror. 


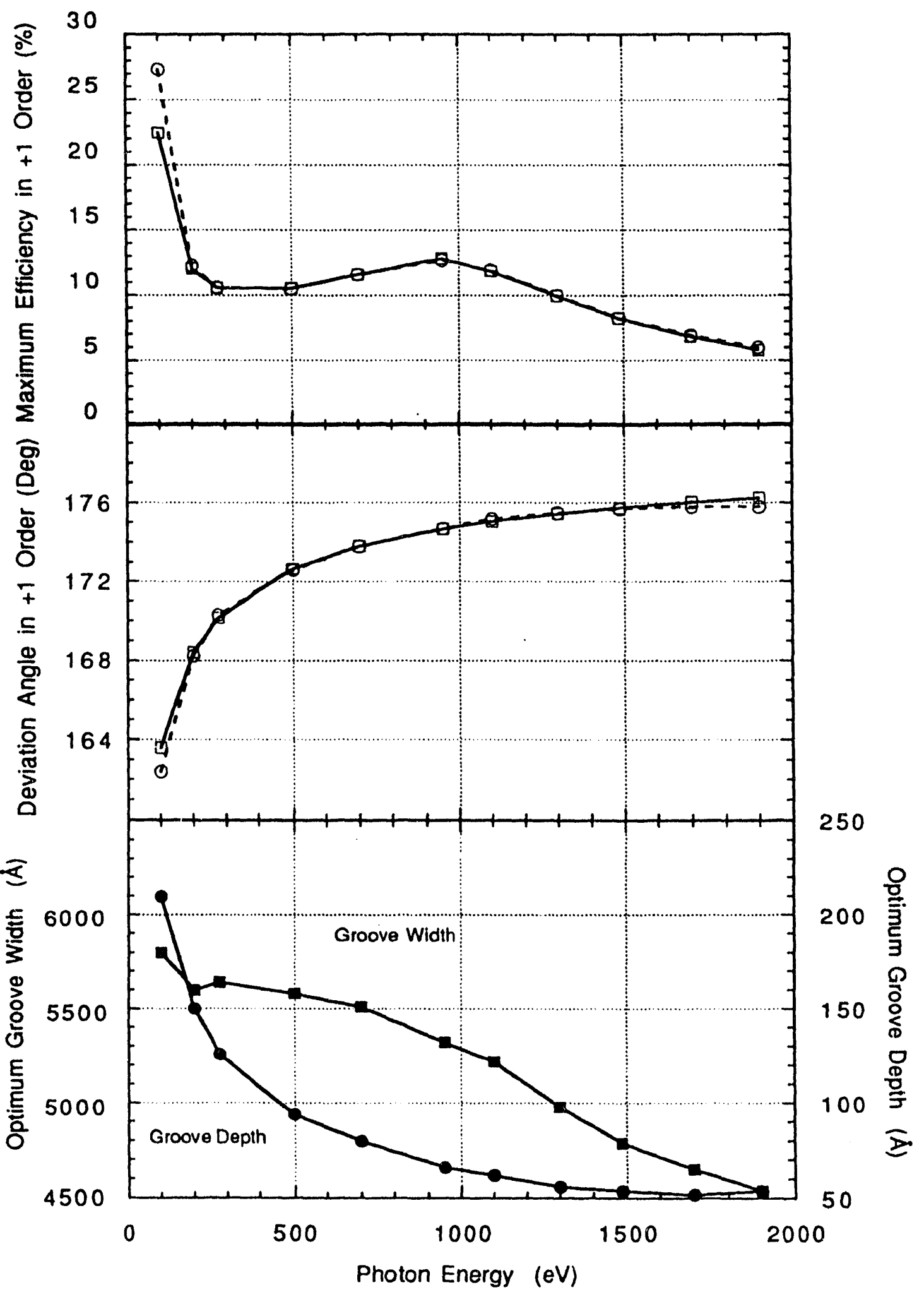

Fig. 10

Theoretical maximum diffraction efficiency is shown for a $1200 \mathrm{l} / \mathrm{mm}$ gold grating for the cases of a free choice of the deviation angle and the groove parameters (dotted) and for the same groove parameters but constraining the deviation angle to follow the fixed plane grating focusing condition with $\mathrm{C}=2.166$ (solid). 


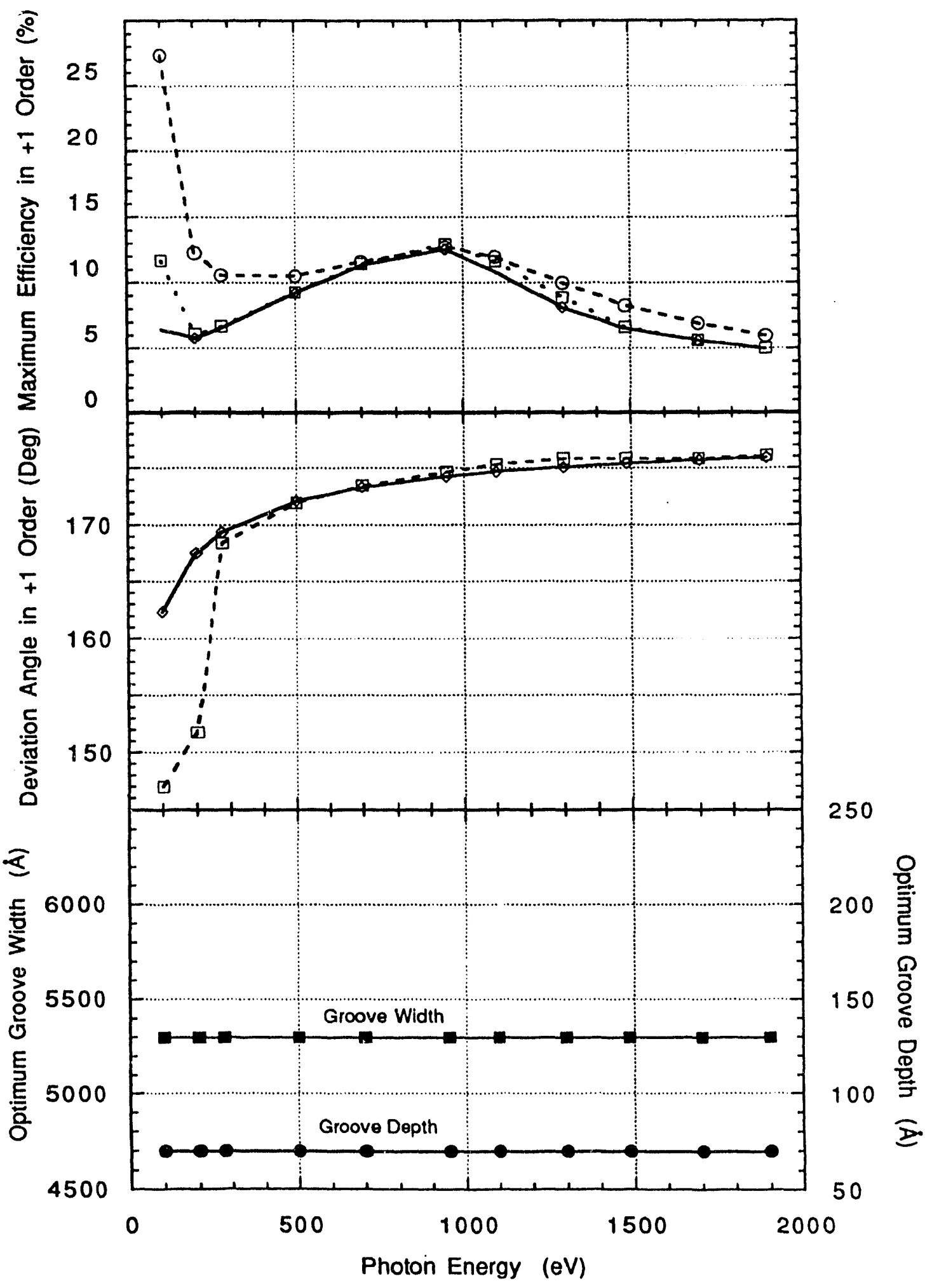

Fig. 11

Theoretical maximum diffraction efficiency is shown for a $1200 \mathrm{l} / \mathrm{mm}$ gold grating for the cases of a free choice of the deviation angle and the groove parameters (long dash), for the case of fixed groove width and depth but a free choice of the deviation angle (short dash) and for the case of fixed groove width and depth but with the deviation angle constrained by $C=$ 2.094 (solid). 

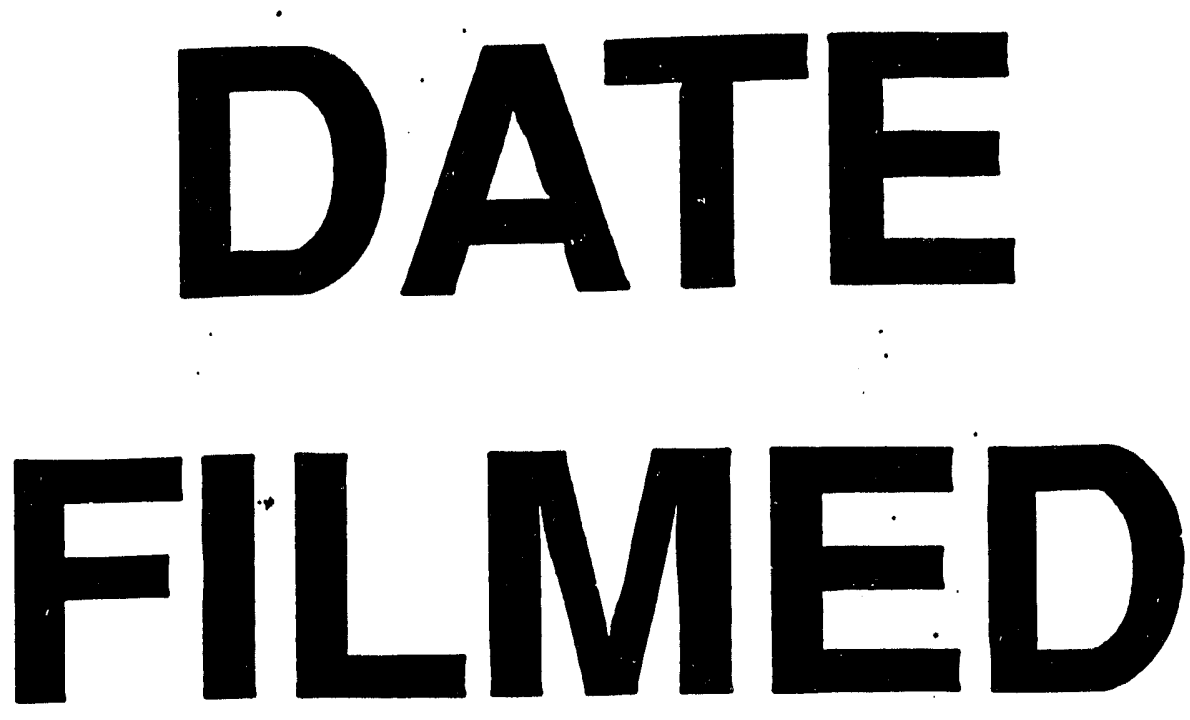

$1 / 21 / 94$
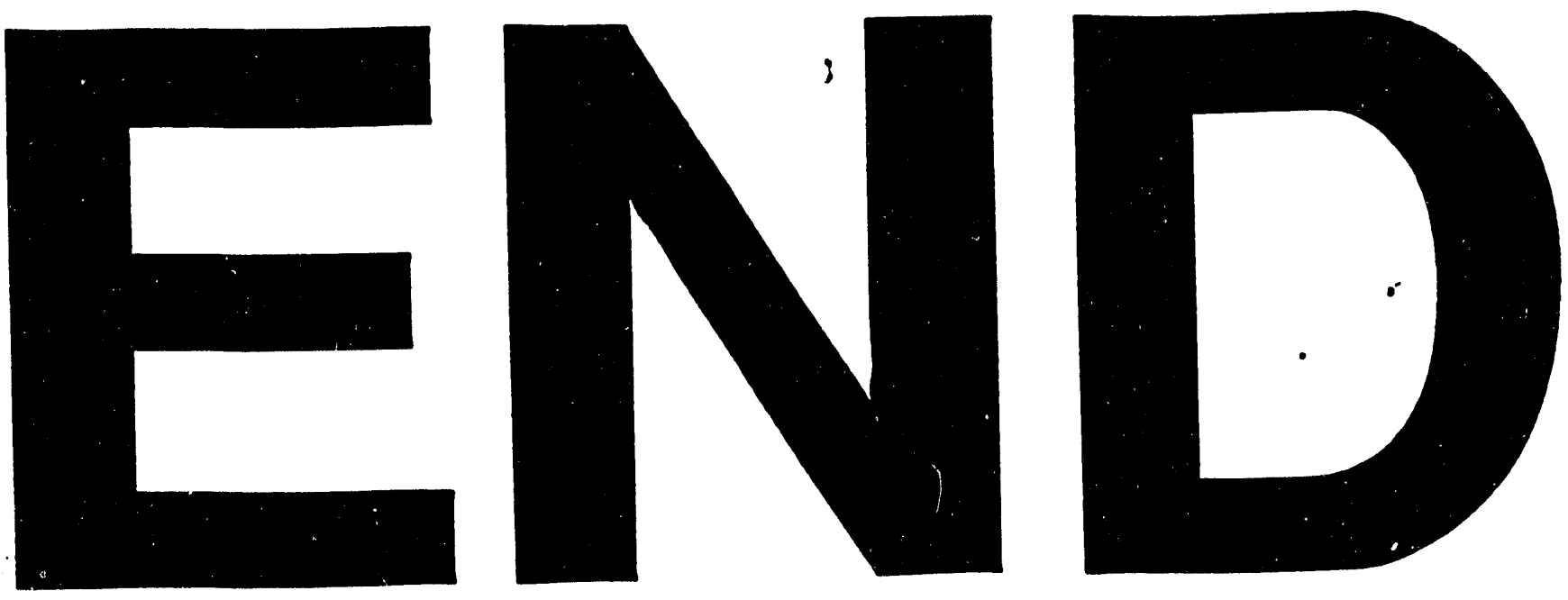


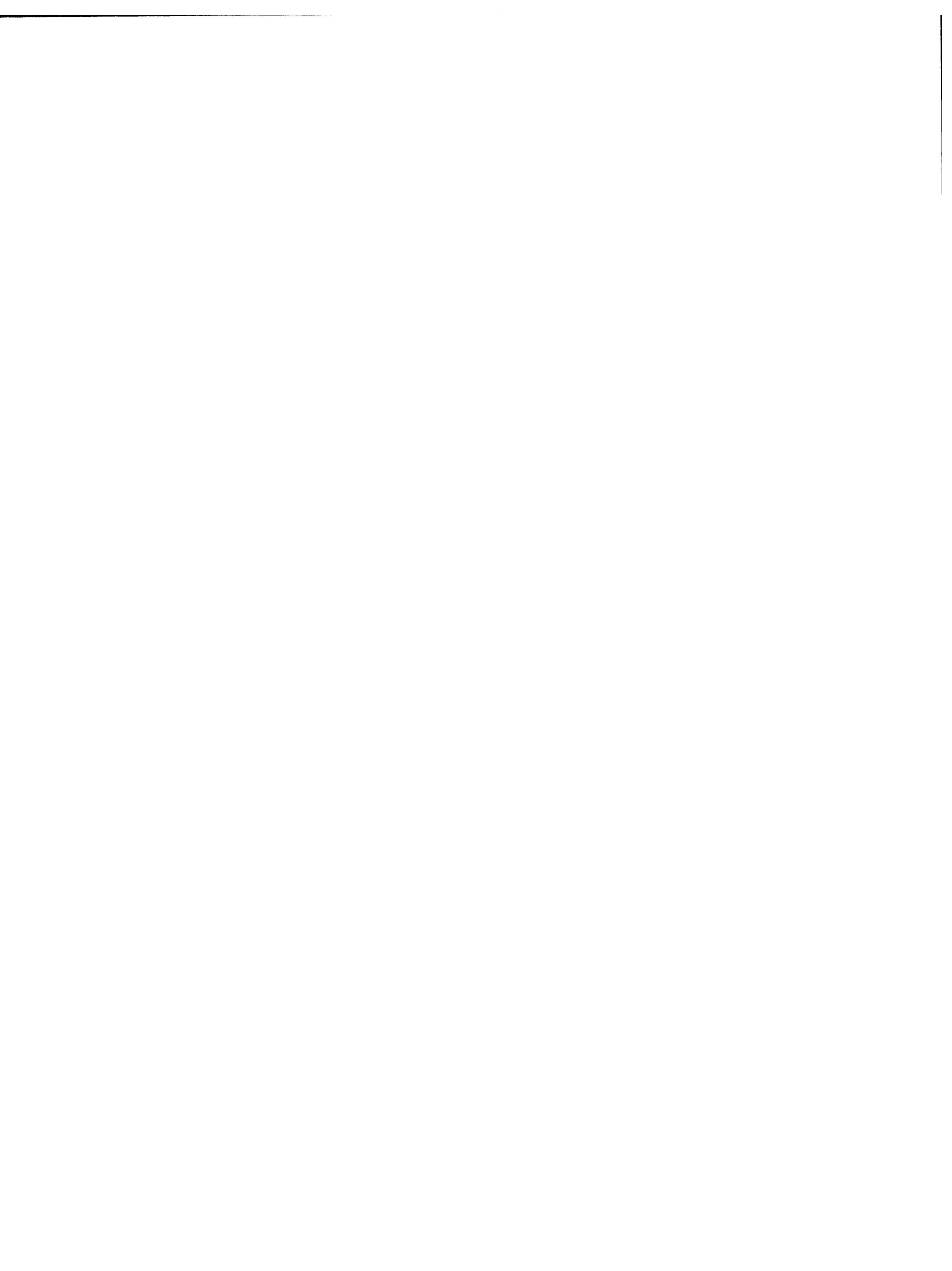

